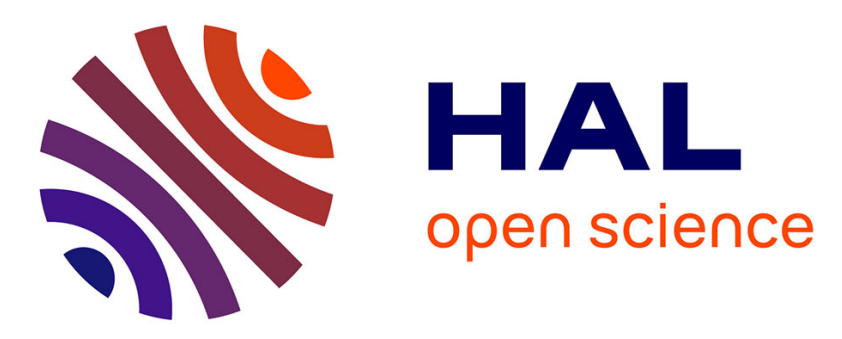

\title{
Simple and energy efficient image compression for pulse-based communication in $\mathrm{THz}$ band
}

\author{
Muhammad Agus Zainuddin, Eugen Dedu, Julien Bourgeois
}

\section{To cite this version:}

Muhammad Agus Zainuddin, Eugen Dedu, Julien Bourgeois. Simple and energy efficient image compression for pulse-based communication in $\mathrm{THz}$ band. International Conference on Advanced Information Networking and Applications, Mar 2017, Taipei, Taiwan. hal-02140346

\section{HAL Id: hal-02140346 \\ https://hal.science/hal-02140346}

Submitted on 27 May 2019

HAL is a multi-disciplinary open access archive for the deposit and dissemination of scientific research documents, whether they are published or not. The documents may come from teaching and research institutions in France or abroad, or from public or private research centers.
L'archive ouverte pluridisciplinaire HAL, est destinée au dépôt et à la diffusion de documents scientifiques de niveau recherche, publiés ou non, émanant des établissements d'enseignement et de recherche français ou étrangers, des laboratoires publics ou privés. 


\title{
Simple and energy efficient image compression for pulse-based communication in $\mathrm{THz}$ band
}

\author{
Muhammad Agus Zainuddin, Eugen Dedu, Julien Bourgeois \\ Univ. Bourgogne Franche-Comté (UBFC) - UFC \\ Institut FEMTO-ST, UMR CNRS 6174, DISC department \\ Numérica, Cours Leprince-Ringuet, BP 21126, 25201 Montbéliard, France \\ E-mail: $\{$ FirstName.LastName $\}$ @ femto-st.fr
}

\begin{abstract}
Terahertz band (0.1-10 THz) provides very large bandwidth, enabling multimedia transmission at short distance. In macro world, ultra broadband communication networks at THz band (TeraNets) provides very large bandwidth for wireless multimedia sensor networks (WMSN). Similarly, recent development in nano-technology (nano-antenna and nano-transceiver) shows that electromagnetic nanocommunications at $\mathrm{THz}$ band support very large bandwidth too, which enables the development of wireless multimedia nano-sensor networks (WMNSN). For both WMSN and WMNSN, the major challenges are simple and energy efficient transmission, since the network consists of a large number of nodes with limited battery capacity. In this paper, we propose a simple, energy efficient and robustness-aware image compression for pulse-based WMSN and WMNSN. We investigate the system performance in terms of image quality, energy efficiency, perpetual operation in nanocommunications and transmission robustness against error. The results show that for these networks, with the trade-off of image quality, the proposed method outperforms JPEG, JPEG 2000, GIF and PNG in all used metrics.
\end{abstract}

\section{INTRODUCTION}

The integration of low-power wireless networking technology and multimedia microelectronics fosters the development of wireless multimedia sensor networks (WMSN) [1]. A node in WMSN has sensing, processing and communication capability to send the information to an end system. In macro scale, WMSN have applications as varied as artificial retina, battlefield surveillance, movement monitoring, volcano monitoring, tsunami or early flood detection [2]. While in micro scale, wireless multimedia nano-sensor networks (WMNSN) allow nano-devices to detect the presence of virus, harmful bacteria and cancer cell in human body [3].

Whether in macro or micro, a node in WMSN has limited resources in transmission range and energy capacity. The main research challenge in WMSN is obtaining energy efficiency to prolong the node lifetime, due to limited battery capacity and difficult process to replace or recharge the battery. Thus, it is essential to reduce the energy consumption whether in computation or in communications process.

In macro scale, wireless sensor networks, energy consumption for transmission (of 1 bit) is considerably larger than com-

This work has been funded by the Ministry of Education and Culture, Indonesia (Ph.D. grant no. 435/E4.4/K/2013), and by Pays de Montbeliard Agglomeration. putation (execution of 1 instruction) [4], [2], [5], [6]. In [4], the authors described that the energy cost to execute 3 million instructions is $3 \mathrm{~J}$, so the computation consumes $1 \mu \mathrm{J} /$ instruction. More recent results show that in computation, conventional microprocessor consumes $1 \mathrm{~nJ} /$ instruction, low-energy digital signal processing (DSP) consumes $0.01 \mathrm{~nJ} /$ instruction and hardwired logic consumes $0.001 \mathrm{~nJ} /$ instruction [5].

As for transmission in macro scale, in [4], the transmission of a $1 \mathrm{kbit}$ packet over a distance of $1 \mathrm{~km}$ using BPSK modulation consumes energy $3 \mathrm{~J}$, which is the same as executing 3 million instructions. Hence, the ratio between transmission and computation is 1:3000. In Ultra-Wide Band (UWB) image transmission system using On-Off Keying (OOK) modulation, radio transmission component consumes $P=15 \mathrm{~mW}$ with rate $R=1.3 \mathrm{Mbps}$ for distance of $4 \mathrm{~m}$ [7]. So, the energy consumption is $E=P / R=11.5 \mathrm{~nJ} / \mathrm{pulse}$, which is three orders of magnitude more than required energy to execute 1 instruction in low-energy DSP. In built-in systems, the energy to send one bit is between 1500 to 2700 for Rockwell WINS nodes and between 220 to 2900 for MEDUSA II nodes bigger than to compute a single instruction [6].

In micro scale (nanonetworks), computation energy in nano-devices remains unknown, since nano-processor using graphene-based nano-transistor is under development [8]. But initial models of energy consumption in nano-devices give a ratio between communication and computation of 10:1 [9], e.g. energy to transmit a pulse is $1 \mathrm{aJ}$ and energy to execute 1 instruction is $0.1 \mathrm{aJ}$. So, in micro scale too, the transmission energy is larger than computation energy.

The previous numbers consider only the transceiver part. However energy consumption for transmission also include the process where central processing unit (CPU) reads the bitstream in memory and gives instructions to signal generator for transmission process, so this process further increases the transmission energy. Moreover, for large transmission distance (e.g. more than 100 meters), transmitter requires power amplifier which consumes much energy. Since nodes in WSN have short transmission range due to the use of low power transmitter, end-to-end transmission is performed in multi-hop fashion. Therefore, total energy consumption for transmission is equal to the sum of transmission energy in each hop from source node to end system. So the transmission energy is 


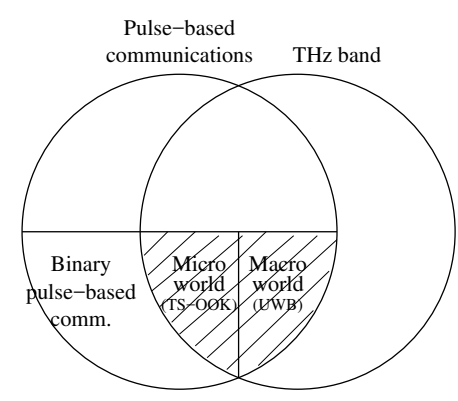

Fig. 1. SEIC application domain.

even greater. Based on all this information, we conclude that computation consumes much less energy than communication. These results motivate the use of compression method before transmission process.

Now we turn our focus on the networks where the compression method can be implemented. Our particular pulsebased communication networks are TeraNets (ultra-broadband communication networks at terahertz band) and nanocommunications. Both TeraNets and nanocommunications will operate at $\mathrm{THz}$ band, which allows transmission rate up to several Tbps [10]. Jornet and Akyildiz [11] proposed the use of $100 \mathrm{fs}$-long pulses transmission by following an asymmetric On-Off Keying modulation spread in time (TS-OOK). In TSOOK, binary 1 represents as a pulse transmission and binary 0 is silence. Pulse duration is smaller than pulse period and time between consecutive bits is fixed.

In pulse-based communication and $\mathrm{THz}$ band communication, the communications can be classified according to the size of devices (scale) as shown in Fig. 1. In micro world, the devices have total dimension of several micro-meter square and the transmission range is below $1 \mathrm{~m}$ [11]. In macro world, the devices are larger and the transmission range is up to $10 \mathrm{~m}$ [12].

In this paper we propose simple and energy efficient image compression (SEIC) for pulse-based communication systems at $\mathrm{THz}$ band. The proposed method can be used in micro and macro scale. SEIC compression is based on discrete wavelet transform (DWT) transformation followed by low weight code.

Compressing an image inevitably consumes energy, hence it is important to compare the energy to transmit an uncompressed image and the energy to compress it and to send the compressed file. As it will be shown later in the article, the energy in uncompressed case is much higher than in compressed case, hence it is better to compress it before transmission. The simulation results show that the proposed method obtain energy efficiency of more than $88 \%$ and outperforms JPEG, JPEG 2000, GIF and PNG in terms of energy efficiency and robustness against transmission error. Our main contributions are:

- We propose an image compression based on DWT transform and NME code, which is simple, energy-efficient and takes into account robustness.

- We compare our proposed method with several existing well known image compression methods such as JPEG, JPEG 200, GIF and PNG.

- We prove that our compression method is useful in WMSN and WMNSN.

- We show that a nano-node is able to perform perpetual image transmission according to state of the art in nanotransceiver and nano-battery.

\section{RELATED WORK}

Since a node in WSNs has limitation in energy capacity, both energy for computation and communication should be minimized. Simple data compression method is necessary to reduce the energy consumption in computation process. In [13], the authors proposed simple data compression for WSN. The method maps the differences of the values between consecutive samples to variable codeword size (output length). The larger the difference, the longer the codeword size. For example, if the difference is \pm 1 the codeword is 010 , and if the difference is \pm 8192 the codeword is 111111111110 . The results show that their method outperforms S-LZW, GZIP and BZIP2 in terms of energy efficiency, a less computational effort and a lower memory occupation. However, the drawback of this method is unreliability. The different value (whether current sample/data is larger of smaller than previous one) is coded with the same codeword, which may lead to estimation error at receiver. The error is propagated even in the absence of transmission error, so perfect reconstruction is almost impossible. In addition, variable codeword sizes require more computational complexity than the constant one. Moreover, the number of bit $1 \mathrm{~s}$ is very large especially in long codewords. Therefore, we conclude that this method is unsuitable in nanocommunications.

In classical WSN, image compression is required to reduce the number of transmission due to the limited bandwidth and limited-battery capacity in sensor devices [2]. Image compression can be classified as lossless and lossy. In general, lossy compression yields higher compression ratio than lossless [14]. Lossless image compression such as PNG and GIF takes the advantages of non-uniform probability distribution for a variable-length codewords. GIF uses Lempel-Ziv-Welch (LZW) code to compress image, while PNG uses deflate code, which is a variant of Lempel-Ziv.

Joint Picture Expert Group (JPEG) and JPEG 2000 are the most popular lossy image compression. JPEG is based on discrete cosine transform (DCT) transform, while JPEG 2000 on discrete wavelet transform (DWT). The results given in [15] show that DWT outperforms DCT in terms of image quality, execution time and transmission robustness, while DCT outperform DWT only in memory usage. Alternatively, memory usage in DWT can be reduced by using block processing like in JPEG [16].

Recently, there are many energy efficient codes for nanonetworks. In [17], the authors investigate the energy efficient code for TS-OOK modulation by taking into account the energy consumption at the receiver. In [18], the authors compare the performance of several low weight codes for nanonetworks 
in terms of energy efficiency, bandwidth expansion, channel capacity, interference reduction and transmission robustness against error.

Our new method is based on DWT transform followed by a low weight code. In image transformation, SEIC uses only the coarse coefficients, i.e. only 1 sub-band from the 4 sub-bands in original DWT. The use of a fixed codeword size in low weight code simplifies the symbol detection in decompression process compared to the variable-length codeword in DCT and DWT.

\section{BACKGROUND}

\section{A. Terahertz propagation model}

In [19], the authors investigate the channel capacity in terahertz band. The results show that terahertz band support for the transmission of several Tbps for small distance. In terahertz band, the electromagnetic propagation is affected by molecular absorption and molecular noise. The path-loss in terahertz band is mainly characterized by the spreading loss and the molecular absorption loss. The spreading loss is the attenuation when electromagnetic wave propagates through the medium, while absorption loss is the attenuation due to absorbed wave's energy by molecules along the transmission path, which converts part of wave energy into internal kinetic energy at the molecule level. The excited molecules re-radiate the absorbed energy, which is modeled as a noise factor. The molecular absorption noise is correlated with the transmitted pulse, where the amount is increased after a pulse transmission and there is background noise unless the molecules are irradiated.

\section{B. TS-OOK modulation}

Jornet et al. [11] proposed the Time-Spread On-Off Keying (TS-OOK) modulation based on very short pulses (one hundred femtosecond-long per Gaussian pulse). For the time being, the only feasible way for electromagnetic nanocommunications is pulse-base modulation. Such pulses have been used in terahertz imaging and biological spectroscopy [20]. During the transmission process, binary 1 is considered as a pulse transmission, while binary 0 as silence (no energy required). The time $T_{s}$ between two consecutive symbols is much longer than the pulse duration $T_{p}$, i.e. $\beta=T_{s} / T_{p} \gg 1$.

\section{C. $N M E$ code}

In TS-OOK modulation, the transmission energy can be reduced by using low weight codes [18]. NME [21] is one such code we have proposed in the past. It obtains energy efficiency by reducing the number of bits 1 in binary sequence. This method uses simple mapping from input symbol to its corresponding codeword (coding table). An example is given in Table I. The most frequent symbols are mapped to codewords with fewer bits 1 . In coding table, input symbols are sorted in decreasing order of their frequency, while codewords are sorted in increasing number of their weight (the number of bits 1 in the codeword). The codewords have the same size (length) as input symbols bits. For codewords with the

\begin{tabular}{ccc}
\hline Input symbol & Symbol frequency & NME \\
\hline 111 & 80 & 000 \\
110 & 70 & 010 \\
101 & 60 & 001 \\
100 & 50 & 100 \\
011 & 40 & 101 \\
010 & 30 & 011 \\
001 & 20 & 110 \\
000 & 10 & 111 \\
\hline
\end{tabular}

TABLE I

THE EXAMPLE MAPPING TABLE FOR NME CODE.

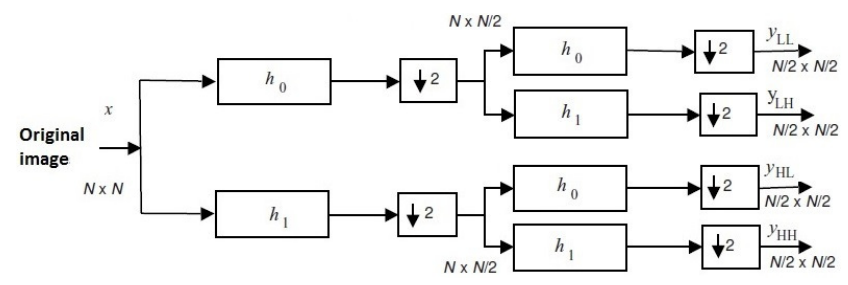

Fig. 2. Computation of a one level DWT decomposition.

same weight, the sorting allows to reduce the number of sequential bits 1 , which is a useful feature in terahertz band communications.

\section{Discrete wavelet transform (DWT)}

Wavelet transform has a high energy compaction which makes it a very suitable candidate for image compression. It also has other properties such as, multi-resolution and progressive reconstruction that provides wavelet a powerful tool for image and video compression [22].

Wavelet-based image compression uses sub-band coding which presents the different frequency components within an image. Sub-band coding consists of a sequence of filtering and sub-sampling processes. The wavelet decomposition for image $x$ is shown in Fig. 2. First, the input sequence $x$ with size $\mathrm{NxN}$ is filtered row by row by two filters (a low pass filter $h_{0}$ and a high pass filter $h_{1}$ ) then sub-sampled by factor 2 , which results in two outputs of length N/2 in each filter. Next, these coefficients are filtered and sub-sampled column by column with the same process as previous (and the same filters). As a result, the output $y$ consists of four DWT coefficients (LL, LH, HL and HH) each of size (N/2) x (N/2). The LL coefficient is called approximation coefficient, while LH, HL and $\mathrm{HH}$ are detail coefficients. The approximation coefficients result in coarse information, which contains the significant information $\left(y_{L L}\right)$. The image compression process discards the insignificant information and maintains the significant information.

In the synthesis or reconstruction stage, the procedures are repeated in reverse direction using another set of low pass and high pass filters (a low pass filter $g_{0}$ and a high pass filter $g_{1}$ ). The reconstructed image has the same size as the original, and the result is close to the original image (but not the same) due to filtering effects. The reconstruction process is shown in Fig. 3. 


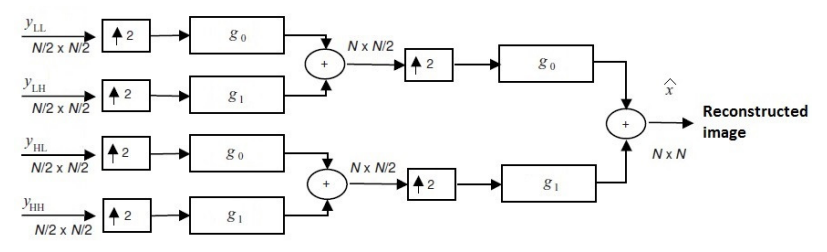

Fig. 3. Computation of a one level DWT reconstruction.

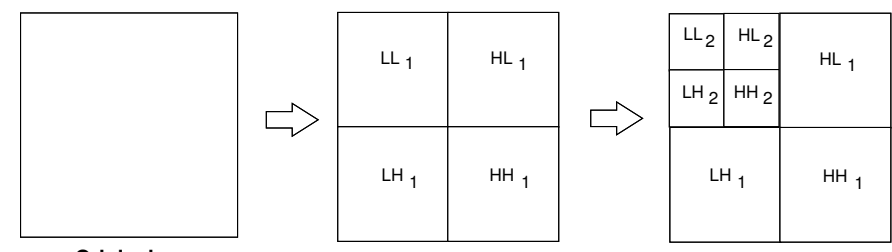

Original

DWT level 1

DWT level 2

Fig. 4. Multi-level DWT transform.

In DWT transform, image is transformed into transform coefficients with the same size of image. Multi-level decomposition can be performed (level $i+1$ ) using sub-band $\mathrm{LL}_{i}$ to produce $\mathrm{LL}_{i+1}, \mathrm{HL}_{i+1}, \mathrm{LH}_{i+1}$ and $\mathrm{HH}_{i+1}$ bands, each with size $\mathrm{N} /(2 \mathrm{i}) \times \mathrm{N} /(2 \mathrm{i})$. The process of multi-level decomposition is illustrated in Fig. 4.

\section{SIMPLE AND ENERGY EFFICIENT IMAGE COMPRESSION (SEIC)}

At the sender, SEIC consists of three steps:

- Perform the first decomposition of DWT (and only this).

- Quantize the coefficients and convert them to binary stream.

- Reduce the number of bit 1 in binary stream by a lowweight code.

These steps are shown in Fig. 5 and detailed in the following algorithm:

1) The image $x$ is filtered row by row using low pass filter $h_{0}$ and down sampled by factor 2 , then filtered column by column using filter $h_{1}$ and down sampled by factor 2 . The output is $y$ with a size of $1 / 4$ of the input $x$.

2) Next, the output of transformation $y$ is quantized by uniform scalar quantizer to fit into $n$ bits pulse code modulation (PCM) or $n$ bit NME code.

3) NME performs simple mapping from input symbol to a codeword, which produces binary stream for transmission.

At receiver, the reconstruction process is performed in reverse direction, i.e., NME decoding [21], de-quantization and DWT inverse transform.

The first step in SEIC algorithm is related to the obtained energy efficiency. As already stated, SEIC uses only one decomposition level. Instead, if several levels are used, the larger the level $i$, the larger the compression ratio which can be obtained. For example, transmitting only $\mathrm{LL}_{1}$ yields compression ratio 4:1 and $\mathrm{LL}_{2}$ yields $16: 1$ as shown in Fig. 4.
Therefore, minimum energy efficiency can be estimated for each level. For example, the minimum energy efficiency using level 1 decomposition is $75 \%$ (this value is obtained from the discard of $75 \%$ coefficients from data input), the minimum energy efficiency for level 2 is $93 \%$ and for level 3 is $98 \%$.

Our method uses only the approximation coefficient and discards the detail coefficients of DWT. SEIC is both simple and energy efficient. It is simple because:

- In a wireless node-device, the compression is performed by an electrical circuit. Compared to other DWT-based image compression methods, such as JPEG 2000, SEIC uses only $25 \%$ of the circuit in DWT transforms. The circuit size is smaller than original DWT (i.e., use only 1 sub-band from the 4 sub-bands), which is preferable for a nano-device.

- Required memory is smaller than original DWT, due to fewer circuit/electrical components. It reduces the amount of required memory by approximately $75 \%$ compared to JPEG 2000.

- Compared to DWT, there is no negative coefficient (negative coefficients appear only in detail coefficients), i.e. there is no additional memory to save negative sign for each of the coefficients.

It is energy efficient because:

- Due to fewer circuit/electrical in DWT components, the computation energy is smaller by approximately $75 \%$ than original DWT.

- Output data size is reduced by $75 \%$ from the input size in each decomposition level, i.e. each decomposition level reduces the number of coefficients by $75 \%$ of data input. As shown in Fig. 4, the input has data size $\mathrm{N} x \mathrm{~N}$, then the approximation coefficient at level $i$ has data size $\mathrm{N} /(2 \mathrm{i})$ $x \mathrm{~N} /(2 \mathrm{i})$. The larger the decomposition level, the larger the energy efficiency (compression ratio) which can be obtained, but the lower the reconstructed image quality.

The use of fixed codeword size in NME decoding makes symbol detection simpler, compared to variable codeword size as used in JPEG and JPEG 2000. In many cases, a 1-bit error in variable codeword size causes symbol error detection for the next symbols (error bits make impossible the reconstruction process).

\section{PERformance ANALYsis}

The goal of this section is to confirm the theory, i.e. computation consumes much less energy than transmission, as given in Introduction, through simulation.

In macro world, the ratio between transmission and computation (10000) is much bigger than in micro world (10), as presented in Introduction, so the energy for computation is negligible compared to transmission. Therefore, in this section we focus on the "worse" case for us, the micro world.

In the following, we numerically investigate the performance of SEIC in terms of visual quality, energy efficiency, perpetual operation and robustness against transmission error, and compare it with several well-known compression 


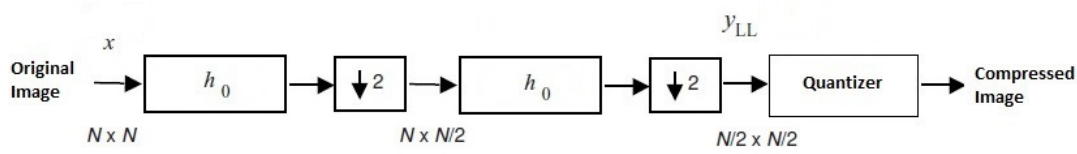

(a)

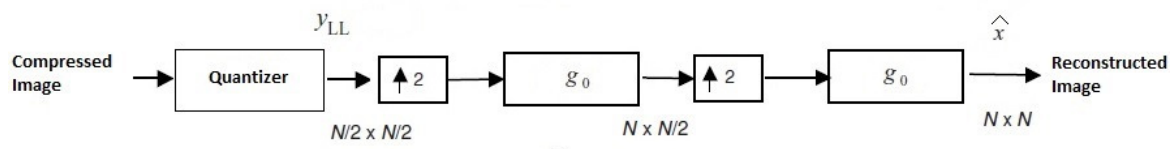

(b)

Fig. 5. Proposed method: (a) SEIC encoder, (b) SEIC decoder

standards (which do not use NME). In order to make our simulation more realistic, we use the following parameters: pulse width $T_{p}=1 \mathrm{ps}$ (the first derivation of a $100 \mathrm{fs}$-long pulse results in a $1 \mathrm{ps}$-long pulse, to prevent DC component in Gaussian pulse [23]), pulse power $P=1 \mu \mathrm{W}$ [24], so pulse energy $E_{p}=P T_{s}=1 \mathrm{aJ}$. In wavelet transform, we use wavelet biorthogonal4.4 and quantization 8. For simulation we use MATLAB. For diversity, in the simulation we use 4 images with resolution $128 \times 128$ pixels, as follows:

- Cancer cell image (cancer128.bmp) to represent an image with micro scale content (a cell).

- Lena image (lena128.bmp) to represent images with high correlation between adjacent pixels.

- Barbara image (barbara128 . bmp) to represent images with moderate correlation between adjacent pixels.

- Baboon image (baboon128.bmp) to represent images with low correlation between adjacent pixels.

\section{A. Image quality}

The metric we used to measure the visual quality of reconstructed images are structural similarity (SSIM) and peak signal to noise ratio (PSNR). SSIM provides results more similar to human visual perception than PSNR [25]. For both of them, the larger the value the closer the received image to the transmitted one. SSIM and PSNR for all images are shown in Table II. GIF and PNG have mean SSIM 1 and PSNR $\infty \mathrm{dB}$, which means perfect reconstruction. For lossy compression, JPEG 2000 has the largest PSNR and SEIC has the lowest PSNR for all images. As shown in Fig. 6, the SEIC image is not perfect, but is sufficiently good. This is the price to pay in order to have a simple and energy efficient compression.

\section{B. Energy efficiency}

In order to investigate the effectiveness of our compression method from energy point of view, we compare the energy in both cases, i.e. the energy to transmit the uncoded image to the energy to compress it and transmit the compressed image:

$$
\begin{gathered}
E_{\text {cons }}^{\text {Uncoded }}=E_{t x}^{\text {Uncoded }} \\
E_{\text {cons }}^{\text {Coded }}=E_{\text {comp }}+E_{\mathrm{tx}}^{\text {Coded }}
\end{gathered}
$$

where $E_{\text {cons }}$ is the energy consumption, $E_{\mathrm{tx}}$ the transmission energy, and $E_{\text {comp }}$ the compression energy. We present the
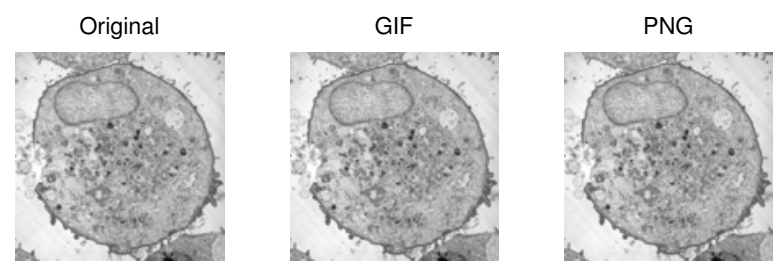

Mean SSIM = 1
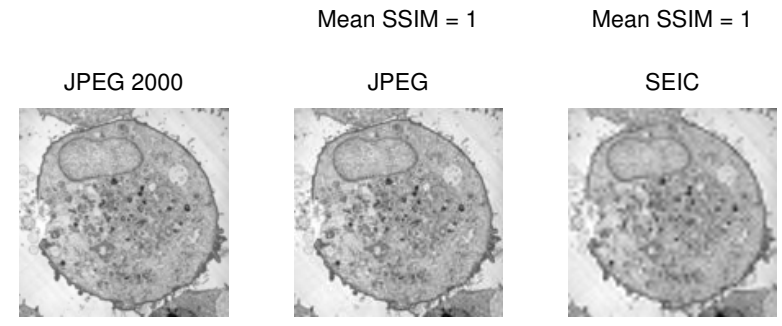

Mean SSIM $=0.99952$

Mean SSIM $=0.93419$

Mean SSIM $=0.82825$

energy on the transmitter, but similar formulas apply for receiver too.

Transmission energy. In TS-OOK modulation, the transmission energy can obtained from the number of transmitted bits 1 and pulse energy:

$$
\begin{aligned}
E_{\mathrm{tx}}^{\text {Uncoded }} & =N_{1}^{\text {Uncoded }} E_{p} \\
E_{\mathrm{tx}}^{\text {Coded }} & =N_{1}^{\text {Coded }} E_{p}
\end{aligned}
$$

where $E_{p}$ is the pulse energy $(1 \mathrm{aJ})$ and $N_{1}$ the number of bits 1. Table II presents the transmission energy for all used methods using these formulas. Column Energy consumption on transmitter shows that the energy varies between approximately 7,000 and 76,000 aJ.

Computation energy for coded. For image with resolution 256x256 pixels, JPEG compression executes 10 million instructions on an 8-bit micro-controller. The computation energy can be reduced by factor 100 using JPEG dedicated hardware [26]. As mentioned before, the computation in a nano-device consumes 0.1 aJ [9] per instruction. So, JPEG hardware compression consumes $100 \mathrm{fJ}$. Therefore, for an image of resolution $128 \times 128$ pixels and processed on a 32-bit 


\begin{tabular}{llrrccc}
\hline Image & Method & $\begin{array}{r}\text { Energy cons. (aJ) } \\
\text { Transmitter }\end{array}$ & $\begin{array}{c}\text { Receiver } \\
\text { eff. }(\%)\end{array}$ & $\begin{array}{c}\text { PSNR } \\
(\mathrm{dB})\end{array}$ & SSIM \\
\hline Cancer & BMP & 76223 & 139696 & - & - & - \\
Cancer & GIF & 64339 & 153024 & 15.6 & $\infty$ & 1 \\
Cancer & PNG & 51080 & 99584 & 33.0 & $\infty$ & 1 \\
Cancer & JPEG 2000 & 47895 & 96520 & 37.2 & 62.0 & 0.99 \\
Cancer & JPEG & 17852 & 36024 & 76.6 & 38.1 & 0.93 \\
Cancer & DWT approx & 16229 & 36992 & 78.7 & 33.1 & 0.83 \\
Cancer & SEIC & 7198 & 36992 & 90.6 & 33.1 & 0.83 \\
\hline Lena & BMP & 65594 & 139696 & - & - & - \\
Lena & GIF & 57797 & 146640 & 11.9 & $\infty$ & 1 \\
Lena & PNG & 42842 & 84752 & 34.7 & $\infty$ & 1 \\
Lena & JPEG 2000 & 37267 & 75344 & 45.5 & 60.8 & 0.99 \\
Lena & JPEG & 14781 & 29256 & 78.3 & 40.4 & 0.95 \\
Lena & DWT approx & 13581 & 36992 & 79.3 & 35.1 & 0.90 \\
Lena & SEIC & 7765 & 36992 & 88.2 & 35.1 & 0.90 \\
\hline Barbara & BMP & 66303 & 139696 & - & - & - \\
Barbara & GIF & 59109 & 149936 & 10.9 & $\infty$ & 1 \\
Barbara & PNG & 42196 & 83280 & 36.4 & $\infty$ & 1 \\
Barbara & JPEG 2000 & 36110 & 72472 & 42.9 & 60.9 & 0.99 \\
Barbara & JPEG & 14410 & 28552 & 77.2 & 42.4 & 0.97 \\
Barbara & DWT approx & 13301 & 36992 & 79.9 & 37.2 & 0.94 \\
Barbara & SEIC & 7742 & 36992 & 88.3 & 37.2 & 0.94 \\
\hline Baboon & BMP & 69411 & 139696 & - & - & - \\
Baboon & GIF & 61582 & 155152 & 11.3 & $\infty$ & 1 \\
Baboon & PNG & 47778 & 95104 & 31.2 & $\infty$ & 1 \\
Baboon & JPEG 2000 & 44939 & 90680 & 35.5 & 62.0 & 0.99 \\
Baboon & JPEG & 15530 & 31784 & 77.6 & 39.1 & 0.93 \\
Baboon & DWT approx & 14076 & 36992 & 79.7 & 35.3 & 0.84 \\
Baboon & SEIC & 7082 & 36992 & 89.8 & 35.3 & 0.84 \\
\hline & & & & & & \\
& & & & & \\
& & 759.3 &
\end{tabular}

TABLE II

ENERGY EFFICIENCY FOR ALL USED METHODS (DWT APPROX MEANS DWT WITH ONLY APPROXIMATION COEFFICIENTS).

micro-controller, as in our case, JPEG compression consumes $E_{\text {comp }}=100 \mathrm{fJ} / 16=6,250 \mathrm{aJ}$. Note that DWT transform consumes less energy than DCT [15].

The previous two paragraphs show that the compression process consumes less energy than transmission $(6,250 \mathrm{aJ}$ vs $7000-76000 \mathrm{aJ})$. Thus, simulation results confirm theory, as given in the Introduction.

Energy efficiency. Energy efficiency denotes the ability of code to reduce the energy consumption at transmitter side:

$$
\xi=\frac{E_{\mathrm{tx}}^{\text {Uncoded }}-\left(E_{\mathrm{tx}}^{\text {Coded }}+E_{\mathrm{tx}}^{\text {Comp }}\right)}{E_{\mathrm{tx}}^{\text {Uncoded }}} 100 \%
$$

The energy efficiency for all images in various methods is shown in Table II, where energy for compression has been discarded since it is negligible. The results show that NME code increases the energy efficiency of DWT with only the approximation coefficients. Our proposed method (SEIC) yields more than $85 \%$ energy efficiency.

To conclude, the energy consumption for compression is negligible compared to the energy for transmission. Therefore, it is useful to compress the image before transmitting it. Otherwise said, the energy lost by compressing an image is much lower than the energy gained by sending a compressed image.

\section{Perpetual operation}

The size of an individual nano-device is in the order of a few cubic micrometers [11]. In advanced health monitoring system, nano-devices can be used to detect infectious agents, such as virus and harmful bacteria [27], and sick cells. For example, cancer cells detected [28] at early stage are easier to cure. We believe that this method will revolutionize the way to cure these diseases. A nano-camera captures cell images, then transmits them to the end system for the physician diagnostic. In our application, nano-camera has $128 \times 128$ pixels resolution, so if the pixel size is $1 \mathrm{~nm}$, then the size of nano-camera is around $128 \times 128 \mathrm{~nm}^{2}$, which still fulfills the size requirement for nano-component.

Recently, novel energy harvesting mechanisms have been proposed allowing perpetual (infinite life time) nanonetworks. One such mechanism is described in [29]. The vibrational energy is harvested by exploiting the piezoelectric effect of zinc oxide $(\mathrm{ZnO})$ nanowires. The nano-battery capacity is approximately $800 \mathrm{pJ}$ when the $9 \mathrm{nF}$ nano-capacitor is charged at $0.42 \mathrm{~V}$ generator voltage and the number of vibration cycles is 2500 cycles. For a vibrator of $50 \mathrm{~Hz}$, such as the vent of air conditioner in offices, the time needed to fully charge the nano-battery is 50 seconds.

The energy harvesting rate $E_{\mathrm{hr}}$ can be obtained from the nano-battery capacity $800 \mathrm{pJ}$ divided by time to fully recharge it 50 seconds, which is $16 \mathrm{pJ} / \mathrm{sec}$. The energy to transmit the cancer cell for uncoded is $E_{\mathrm{tx}}=E_{p} N_{1}^{\text {Uncoded }}=73,126 \mathrm{aJ}$. The perpetual operation for uncoded $E_{\mathrm{hr}} / E_{\mathrm{tx}}=16 \mathrm{pJ} /$ $73,126 \mathrm{aJ} \approx 220 \mathrm{images} / \mathrm{second}$. For SEIC, the energy to transmit the cancer cell is $E_{\mathrm{tx}}=E_{p} N_{1}^{\mathrm{SEIC}}=7,198 \mathrm{fJ}$. The perpetual operation for SEIC $E_{\mathrm{hr}} / E_{\mathrm{tx}}=16 \mathrm{pJ} / 7,198 \mathrm{fJ} \approx$ 2,220 images/second.

\section{Robustness against transmission error}

In general, compressed data is vulnerable to transmission error. One bit error may cause error propagation in reconstruction process which destroys the received image, e.g., distorted or file unable to open. This is not the case for SEIC. In this section we present the effect of error bit for transmitted image for all methods.

In order to simulate the transmission at terahertz band, we use HITRAN (HIgh resolution TRANsmission molecular absorption database), an online catalog [30] for path-loss computation. Molecular absorption noise in $\mathrm{THz}$ band is function of distance. Larger distance means larger noise in the received signal, which causes error in detection process. Using the bit error rate (BER) equations from [31], and pulse energy $1 \mathrm{aJ}$, the BER when sending only bits $1\left(B E R_{1}\right)$ and only bits $0\left(B E R_{0}\right)$ are shown in Fig. 7. As expected, it shows that the BER when transmitting bit 1 is always larger than when sending only bit $0\left(B E R_{1} \geq B E R_{0}\right)$.

We investigate here the effect of error bits in the reconstruction (decompression) process at receiver. Due to limited computation in nano-devices, hard decision method is utilized in nano-receiver. Therefore, terahertz band is modeled as a binary asymmetric channel (BAC) and channel output as a discrete random variable [31]. We simulate the transmission at distance $1 \mathrm{~cm}$ and obtain $B E R_{0}=1.7 \times 10^{-8}$ and $B E R_{1}=5.0 \times 10^{-4}$, as shown in Fig. 7. The effect of error 


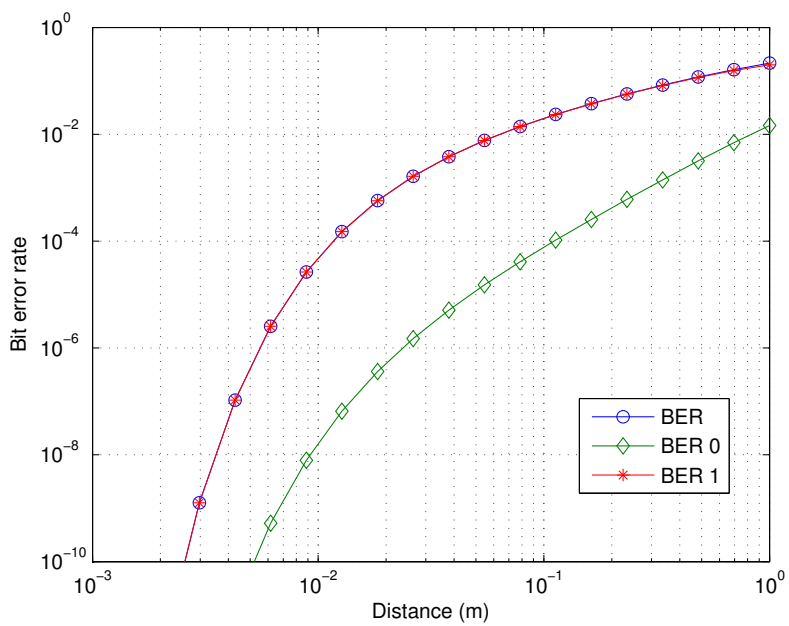

Fig. 7. $B E R_{0}$ and $B E R_{1}$ as function of distance.

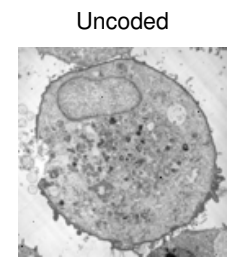

Mean SSIM $=0.99274$

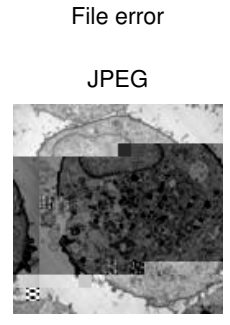

Mean SSIM $=0.46523$

Mean SSIM $=0.19238$

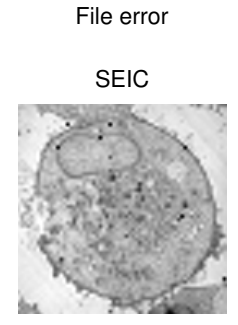

Mean SSIM $=0.81126$

Fig. 8. Visual result of received compressed Cancer image for various methods.

bits in reconstruction process of each compression method are different. Using the same noise, the visual quality of reconstructed image for all methods is shown in Fig. 8, 9, 10, and 11. In uncoded transmission, error bits affect only certain pixels, which is also the same as SEIC method. In JPEG 2000 and JPEG, the error propagates, e.g., one bit error causes errors in many pixels. In GIF and PNG, the error results in an image which cannot be reconstructed (file cannot be opened). In order to obtain reliable transmission for compressed image, GIF, PNG, JPEG and JPEG 2000 require complex (powerful) error correction code, which is impractical for limited computation in nano-devices. While SEIC can use simple error correction code such as Hamming code, since error does not propagate in decompression process. As conclusion, SEIC method is more robust against transmission error.

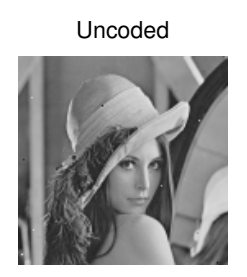

GIF

PNG

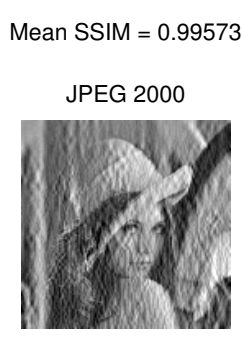

Mean SSIM $=0.47834$

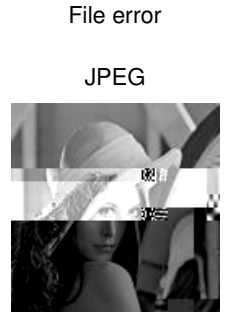

Mean SSIM $=0.3036$

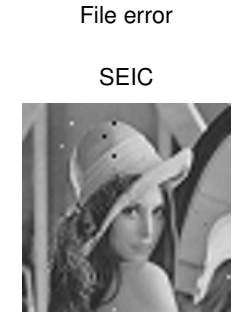

Mean SSIM $=0.87998$
Fig. 9. Visual result of received compressed Lena image for various methods.

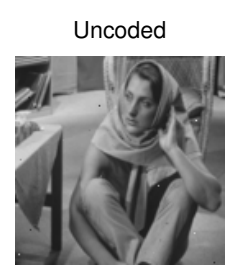

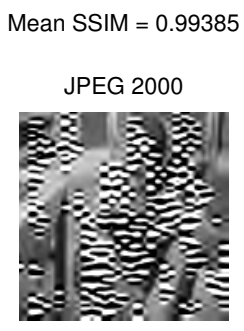

Mean SSIM $=0.25715$
GIF

$P N G$
Fig. 10. Visual result of received compressed Barbara image for various methods.

\section{CONCLusions}

We presented a simple and energy efficient image compression (SEIC) for binary pulse-based wireless sensor networks at terahertz band. The method uses only the approximation coefficients of DWT transform followed by NME code. The simulation results show that our proposed method outperforms JPEG, JPEG 2000, GIF and PNG in several metrics: energy efficiency, perpetual operation in nanocommunications and robustness against transmission error. The trade-off is a lower image quality at receiver.

In future work we will include the computational energy to have a complete knowledge in energy consumption and perform fair comparison between image compression methods. 

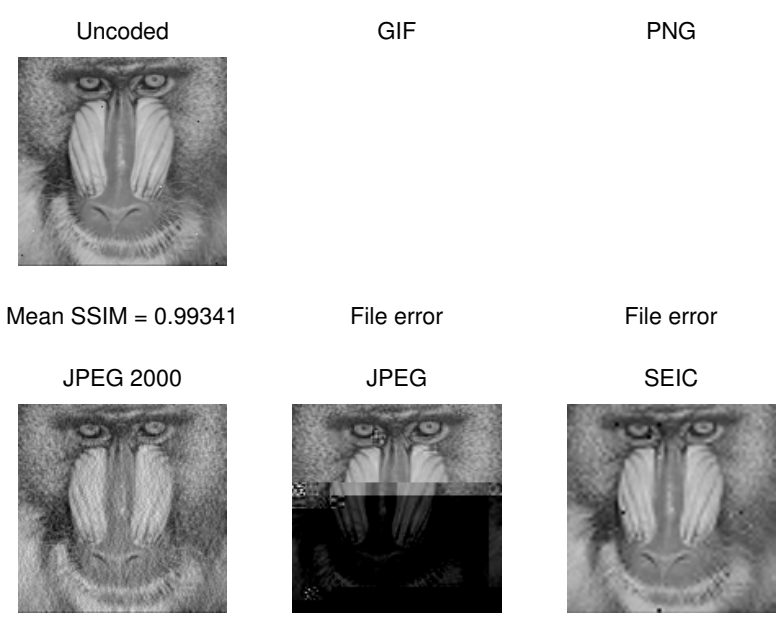

Mean SSIM $=0.64239$

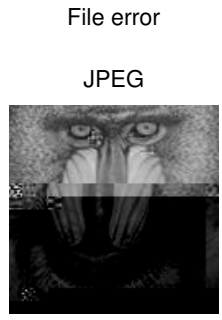

PNG

Mean SSIM $=0.3345$
Mean SSIM $=0.83451$

Fig. 11. Visual result of received compressed Baboon image for various methods.

\section{REFERENCES}

[1] I. F. Akyildiz, T. Melodia, and K. R. Chowdhury, "A survey on wireless multimedia sensor networks," Computer Networks, vol. 51, pp. 921-960, Mar. 2007.

[2] I. F. Akyildiz and M. C. Vuran, Wireless Sensor Networks. John Wiley \& Sons Ltd., 2010.

[3] J. M. Jornet and I. F. Akyildiz, "The internet of multimedia nano-things," Nano Communication Networks, vol. 3, no. 4, pp. 242-251, Dec. 2012.

[4] G. Pottie and W. Kaiser, "Wireless integrated network sensors," Communications of the ACM, vol. 43, no. 5, pp. 51-58, May 2000.

[5] M. Belleville and C. Condemine, Energy Autonomous Micro and Nano Systems. Willey-ISTE, 2012.

[6] H. Karl and A. Wilig, Protocols and Architectures for Wireless Sensor Networks. Wiley, 2007.

[7] S. Chen, W. Tang, X. Zhang, and E. Culurciello, "A 64 x 64 pixels UWB wireless temporal-difference digital image sensor," IEEE Transactions on VLSI Systems, vol. 20, no. 12, pp. 2232-2240, Dec. 2012.

[8] M. C. Lemme, "Current status of graphene transistors," Solid State Phenomena, vol. 156-158, pp. 499-509, Nov. 2009.

[9] N. Akkari, J. M. Jornet, P. Wand, E. Fadel, L. Elrefaie, M. G. A. Malik, S. Almasri, and I. F. Akyildiz, "Joint physical and link layer error control analysis for nanonetworks in the terahertz band," Wireless Networks, pp. 1-13, Aug. 2015.

[10] J. M. Jornet and I. F. Akyildiz, "Channel modeling and capacity analysis for electromagnetic wireless nanonetworks in the terahertz band," IEEE Transactions on Wireless Communications, vol. 10, no. 10, pp. 32113221, Oct. 2011.

[11] _ - "Femtosecond-long pulse-based modulation for terahertz band communication in nanonetworks," IEEE Transactions on Communications, vol. 62, no. 5, pp. 1742-1754, May 2014.

[12] I. F. Akyildiz, J. M. Jornet, and C. Han, "Terahertz band: Next frontier for wireless communications," Physical Communication, vol. 12, pp. 16-32, Sep. 2014.

[13] F. Marcelloni and M. Vecchio, "Simple algorithm for data compression in wireless sensor networks," IEEE Communications letters, vol. 12, no. 6, pp. 411-413, Jun. 2008.

[14] J.-N. Hwang, Multimedia Networking: from theory to practice. Cambridge university press, 2009.

[15] O. Ghorbel, W. Ayedi, M. W. Jmal, and M. Abid, "DCT \& DWT images compression algorithms in wireless sensor networks: comparative study and performance analysis," International Journal on Wireless \& Mobile Networks, vol. 4, no. 6, pp. 45-59, Dec. 2012.

[16] M. Angelopoulou, K. Masselos, P. Cheung, and Y. Andreopoulous, "A comparison of 2-d discrete wavelet transform computation schedules

on FPGAs," in IEEE Field Programmable Technology (FTP), Bangkok, Thailand, Dec. 2006, pp. 181-188.

[17] L. Huang, W. Wang, and S. Shen, "Energy-efficient coding for electromagnetic nanonetworks in the terahertz band," Ad Hoc Networks, vol. 40 , pp. 15-25, Jan. 2016.

[18] M. A. Zainuddin, E. Dedu, and J. Bourgeois, "Low-weight code comparison for electromagnetic wireless nanocommunications," IEEE Internet of Things, vol. 3, pp. 38-48, Feb. 2016.

[19] J. M. Jornet and I. F. Akyildiz, "Information capacity of pulse-based wireless nanosensor networks," in IEEE Communications Society Conference on Sensor, Mesh and Ad Hoc Communications and Networks (SECON), ser. 8. Salt Lake City, Utah, USA: IEEE, Jun. 2011, pp. 80-88.

[20] D. Woolard, P. Zhao, C. Rutherglen, Z. Yu, P. Burke, S. Brueck, and A. Stintz, "Nanoscale imaging technology for THz-frequency transmission microscopy," International Journal of High Speed Electronics and Systems, vol. 18, no. 1, pp. 205-222, Mar. 2008.

[21] M. A. Zainuddin, E. Dedu, and J. Bourgeois, "Nanonetwork minimum energy coding," in IEEE International Conference on Ubiquitous Intelligence and Computing (UIC), ser. 11, Bali, Indonesia, Dec. 2014, pp. 96-103.

[22] K. S. Thyagarajan, Still image and video compression with Matlab. John Wiley \& Sons, Inc., 2011.

[23] M. A. Zainuddin, E. Dedu, and J. Bourgeois, "The effects of nanosensors movements on nanocommunications," in The 2nd ACM Nanocom, Boston, Massachusetts, USA, Sep. 2015, pp. 1-6.

[24] D. Dragoman and M. Dragoman, "Terahertz fields and applications," Progress in Quantum Electronics, vol. 28, no. 1, pp. 1-66, 2004.

[25] Z. Wang, A. Bovik, H. Sheikh, and E. Simoncelli, "Image quality assessment: from error visibility to structural similarity," IEEE Transaction on Image Processing, vol. 13, no. 4, pp. 600-612, Apr. 2004.

[26] V. G. Oklobdzija, Digital Design and Fabrication. CRC Press, Taylor and Francis Group, 2007.

[27] P. Tallury, A. Malhotra, L. M. Byrne, and S. Santra, "Nanobioimaging and sensing of infectious diseases," Advanced Drug Delivery Reviews, vol. 62, no. 4, pp. 424-437, Mar. 2010.

[28] I. E. Tothill, "Biosensors for cancer markers diagnosis," Seminars in Cell \& Developmental Biology, vol. 20, no. 1, pp. 55-62, 2009.

[29] J. M. Jornet and I. F. Akyildiz, "A joint energy harvesting and communication analysis for perpetual wireless nanosensor networks in the terahertz band," IEEE Transactions on Nanotechnology, vol. 11, no. 3, pp. 570-580, May 2012.

[30] L. Rothman, I. Gordon, A. Barbe, D. Benner, P. Bernath, M. Birk, V. Boudon, L. Brown, and A. Campargue, "The HITRAN 2008 molecular spectroscopic database," Quantitative Spectroscopy and Radiative Transfer, vol. 110, no. 9-10, pp. 533-572, Jun. 2009.

[31] J. M. Jornet, "Low-weight error-prevention codes for electromagnetic nanoneworks in the terahertz band," Nano Communications Networks, vol. 5, no. 1-2, pp. 35-44, May 2014. 